\title{
Curiosity and susceptibility to cigarette smoking among cigarette-naïve, waterpipe smoking US youth: National Youth Tobacco Survey, 2014
}

\author{
Roed Boheloh'
}

\begin{abstract}
INTRODUCTION Waterpipe smoking is harmful and addictive and can be a gateway to cigarette smoking. This study examined the association between curiosity and susceptibility to cigarette smoking among cigarette-naïve US youth who smoke waterpipe using cross-sectional data from the National Youth Tobacco Survey, 2014.

METHODS Susceptibility to cigarettes was assessed using a composite index of three questions: 'Do you think you will smoke a cigarette in the next year?', 'Do you think that you will try a cigarette soon?', and 'If one of your best friends were to offer you a cigarette, would you smoke it?'. Curiosity was assessed by one question: 'Have you ever been curious about smoking a cigarette?'.

RESULTS Among current waterpipe smokers, 48.5\% (95\% CI: 43.1-53.9) were susceptible to cigarettes and 42.6\% (95\% CI: 36.5-48.9) were curious about cigarettes. Overall, 45.1\% (95\% CI: 38.9-51.4) and 46.5\% (95\% CI: 40.2-52.8) of those living with a waterpipe smoker were susceptible and curious about cigarettes, respectively. Current waterpipe smokers had significantly higher odds of susceptibility to cigarettes (aOR=1.5, 95\% CI: 1.1-2.3, $\mathrm{p}<0.04)$, but not for curiosity about cigarettes $(\mathrm{aOR}=1.2,95 \% \mathrm{CI}: 0.83-1.70, \mathrm{p}=0.3)$.

concLusions Initiation of cigarettes among adolescents who smoke waterpipe can be due to increasing susceptibility to cigarette smoking rather than increasing curiosity about cigarettes.
\end{abstract}

\author{
AFFILIATION \\ 1 Department of Epidemiology, \\ Robert Stempel College of \\ Public Health and Social Work, \\ Florida International University, \\ Miami, United States

\section{CORRESPONDENCE TO} \\ Raed Bahelah. Florida \\ International University, \\ 15467 SW 86 Terrace, 33193 \\ Miami, United States. \\ E-mail: rbahelah@gmail.com
}

\section{KEYWORDS}

waterpipe, cigarettes, susceptibility, curiosity, NYTS

Received: 5 August 2017

Revised: 24 October 2017

Accepted: 11 November 2017

\section{INTRODUCTION}

Although the prevalence of cigarette smoking among youth in the US has declined, it is estimated that one in four high school students is a current cigarette smoker ${ }^{1}$. The majority of smokers initiate smoking during adolescence, and most young smokers continue to smoke during adulthood ${ }^{1}$. Thus, adolescence and early adulthood represent critical periods for susceptibility to and uptake of cigarettes, and other tobacco products ${ }^{2}$. Despite the existence of policies for the prevention of smoking initiation, each day more than 2000 youths in the US become daily cigarette smokers ${ }^{3}$. The use of non-cigarette tobacco products, such as the waterpipe (hookah, shisha), is increasing worldwide, mainly among youth and young adults ${ }^{4}$. A recent report from the CDC (2014) has shown that among a nationally representative sample of adolescents, $9.4 \%$ and $9.2 \%$ of high school students smoked waterpipe and cigarettes, respectively, in the past 30 days $^{5}$. In addition, waterpipe is increasingly becoming the first tobacco product tried by youth ${ }^{6}$, and different studies have shown that cigarette-naïve youth who smoke waterpipe are at a higher risk of initiating cigarette smoking ${ }^{7-11}$.

Susceptibility and curiosity are two commonly used measures to identify cigarette-naïve youth at risk of experimenting with cigarettes ${ }^{11,12}$. Both susceptibility and curiosity are based on a lack of firm negative response against interest or cognitive readiness to smoke cigarettes ${ }^{11,12}$, and each independently predicts experimentation with cigarettes ${ }^{12}$. For example, a 6-year longitudinal study among 13-15 year-old never-smokers in the US found that adolescents who were definitely not curious about cigarettes, at baseline, were less likely to experiment with cigarettes at follow up, compared with those who were potentially or definitely curious about cigarettes ${ }^{13}$. Previous research has focused on the association between exposure to 
pro-tobacco ads and curiosity and susceptibility to cigarettes ${ }^{13,14}$. However, the availability of non-cigarette tobacco products might increase youth curiosity and/or susceptibility to experiment with cigarettes, beyond the exposure to tobacco ads.

Using a representative sample of US youth, from the 2014 National Youth Tobacco Survey (NYTS), this study examined the association between current waterpipe use, and curiosity and susceptibility to cigarette smoking among US middle and high school adolescents who never smoked cigarettes. Examining the association between waterpipe use and curiosity and susceptibility to cigarettes, separately, is important for understanding which mechanism (curiosity vs susceptibility) underlies the proposed gateway hypothesis for cigarette smoking among cigarette-naïve waterpipe smokers.

\section{METHODS}

\section{Dataset and study population}

Data come from the 2014 NYTS; a school-based, selfadministered survey among a large, nationally representative sample of middle (6-8 grades) and high (9-12 grades) school US adolescents from public and private schools ${ }^{15}$. In 2014, the NYTS inquired about ever and current (past 30 days) use of nine tobacco products (cigarettes; cigars, cigarillo, or little cigars; chew tobacco, snuff, or dip; electronic cigarettes; waterpipe; pipe; bidis; snus; and dissolvable tobacco). Information about student socio-demographics, smoking behaviors, attitudes, and exposure to tobacco ads were collected $^{16}$. A stratified, three-stage cluster sampling design was used by the NYTS to obtain a representative sample of US middle and high school adolescents. The sampling design includes primary sampling units (PSUs) within each stratum, schools within each selected PSU, and in the final stage, classes within each selected school ${ }^{15,16}$. More details about NYTS are available elsewhere ${ }^{16}$.

The sample for this study included adolescents who never smoked cigarettes, not even a puff or two. This was assessed by the survey question: 'Have you ever tried cigarette smoking, even one or two puffs?'. Adolescents who answered 'No' to this question were considered never smoked cigarettes and were included in the analysis $(\mathrm{N}=16,709$; weighted \%= 76.39). Since the data are publicly available, this study deemed exempt from IRB approval.

\section{Outcome variables}

Two outcomes, curiosity about cigarettes and susceptibility to cigarette smoking, were studied. Each of these outcomes independently predicts experimentation with cigarettes ${ }^{12}$. Susceptibility to cigarette smoking was assessed by a composite index using three survey questions: 'Do you think you will smoke a cigarette in the next year?', 'Do you think that you will try a cigarette soon?', and 'If one of your best friends were to offer you a cigarette, would you smoke it?. Each question has four response options: 'Definitely yes', 'Probably yes', 'Probably not', and 'Definitely not'. Students who answered 'Definitely not' to all three questions were considered not susceptible to cigarette smoking and those who gave a response other than 'Definitely not' to any of the three questions were considered susceptible to cigarette smoking ${ }^{13}$. Curiosity was assessed by the survey question: 'Have you ever been curious about smoking a cigarette?', with four response options: 'Definitely yes', 'Probably yes', 'Probably not', and 'Definitely not'. Students who answered 'Definitely not' were considered not curious, and curious if answered otherwise ${ }^{14,17}$.

\section{Explanatory variable}

The primary independent variable is current waterpipe smoking, defined as smoking waterpipe at least once in the past 30 days ${ }^{13}$. Eight hundred (3.33\%) participants have missing data on current waterpipe smoking and were excluded from the analysis. Thus, the final sample included 1,349 (6.17\%) current waterpipe smokers.

\section{Covariates}

Selection of covariates was guided by the socio-environmental framework of smoking initiation among adolescents, and by reviewing the literature for factors related to curiosity and susceptibility to cigarette smoking or smoking initiation among youth. Covariates included were socio-demographics, current use of other tobacco/nicotine products (electronic cigarettes, cigar/ cigarillo/little cigar, smokeless tobacco), exposure to secondhand smoke/vape, exposure to pro-tobacco ads, receptivity to tobacco marketing, and beliefs about smoking-related harm ${ }^{13,15,17,18}$.

Current use of tobacco/nicotine was defined as the use of a tobacco/nicotine product on at least one day during the past 30 days. Smokeless tobacco was defined as the use of chewing tobacco, snuff, dip, snus, or dissolvable tobacco ${ }^{19}$. To assess exposure to secondhand smoke (from a waterpipe) or vape (from electronic cigarettes), students were asked if anyone who lives with them now uses either of these products.

Exposure to pro-tobacco ads in convenient stores was assessed by the question: 'When you go to a convenience store, supermarket, or gas station, how often do you see ads or promotions for cigarettes or other tobacco products?; on the internet by the question: "When you are using the internet, how often do you see ads or promotions for cigarettes or other tobacco products?; in magazines/newspapers by the question 'When you read newspapers or magazines, how often do you 
see ads or promotions for cigarettes or other tobacco products?'; and in movies by the question 'When you watch TV or go to the movies, how often do you see actors and actresses using cigarettes or other tobacco products?'. For all these variables, the exposure was classified into never, rarely/sometimes, and most of the time/always. Receptivity to tobacco marketing was assessed by the question: 'How likely is it that you would ever use or wear something - such as a T-shirt, hat or sunglasses - with a tobacco brand name, logo, or picture on it?'. Students who answered 'Very likely' or 'Somewhat likely' were classified as having high receptivity and those who answered 'Somewhat unlikely' or 'Very unlikely' were classified as having low receptivity ${ }^{14}$.

Two questions were used to assess students' beliefs about smoking-related harm: 'How much do you think people harm themselves when they smoke cigarettes some days but not every day?', and 'How strongly do you agree with the statement "All tobacco products are dangerous"?? Responses to the first question were classified into no harm, little/some harm, and a lot of harm. The second question has four responses: strongly agree, agree, disagree, and strongly disagree. Agree and strongly agree were grouped under the category 'agree', while disagree and strongly disagree were grouped under the category 'disagree'.

\section{Statistical analysis}

Descriptive statistics for the sample's characteristics were calculated and presented by curiosity and susceptibility status using percentages and 95\% confidence intervals (CIs). All percentages were weighted using sampling weights to account for the complex survey design and non-response ${ }^{20}$. In the bivariate analysis, percentages were considered statistically different when their corresponding CIs were not overlapping ${ }^{21}$. Participants with missing values in the outcome variables were excluded: 64 (0.33\%) were missing curiosity status, while 54 $(0.25 \%)$ were missing susceptibility status. Missing values in the covariates were excluded as they were overall low and ranged from $0.61 \%$ in age groups, to $3.33 \%$ in current use of smokeless tobacco products. Consequently, the percentages throughout this paper are for those with complete data. Separate multivariable logistic regression models were used to examine the factors associated with each outcome (curiosity, susceptibility) and all factors were entered simultaneously into these models. Hosmer-Lemeshow test was used to evaluate the model's goodness-of-fit, and a p-value $>0.05$ indicates a good model fit ${ }^{22}$. Adjusted odds ratios (aOR) with 95\% CIs were reported and standard errors were estimated using Taylor linearization method. All analyses were performed using svy command in Stata 13 (StataCorp, College Station, Texas) and the level of significance was set at $\mathrm{p}<0.05$.

\section{RESULTS}

\section{Somple characteristics}

The sample for this study consists of 16,709 US youths who never smoked a cigarette, not even a puff or two. Among them $27.61 \%$ (95\% CI: 26.47-28.78) were susceptible to cigarettes and $30.05 \%$ (95\% CI: 28.91-31.21) were curious about cigarette smoking. Table 1 presents the sample characteristics. There were no significant differences in the percentage of boys and girls who were curious or susceptible to cigarette smoking. Youth age groups, those aged 17 years or more were less susceptible to cigarettes (24.16\%, 95\% CI: 21.51-27.03). Hispanic youth were more susceptible to cigarette smoking than all other races/ ethnicities (Table 1).

\section{Relationship between curiosity and susceptibility to cigarettes}

Among the whole sample, 1736 youths (9.95\%, 95\% CI: 9.3210.58 ) were not curious but susceptible to cigarettes, compared with 3060 youths (17.84\%, 95\% CI: $16.85-18.82)$ who were both curious and susceptible to cigarettes.

\section{Bivariate analysis}

Susceptibility to cigarette smoking was higher among current waterpipe smokers and among individuals who were living with a waterpipe smoker than those who were not waterpipe smokers or living with someone who smokes waterpipe. Similar findings were found for electronic cigarettes, cigar/cigarillo/little cigar, or smokeless tobacco. Students exposed to pro-tobacco ads at convenient stores, internet, newspapers/magazines, or movies, and those highly receptive to tobacco marketing were more susceptible to cigarette smoking. Students who agree that all tobacco products are dangerous or believe people harm themselves a lot when they smoke somedays were less susceptible to cigarette smoking.

A higher percentage of current users of waterpipe or electronic cigarettes, but not cigar/cigarillo/little cigar or smokeless tobacco, were curious about cigarettes than non-users. Students living with someone who uses waterpipe, cigarettes, or electronic cigarettes were more curious about cigarettes than those who did not. Compared to those never exposed to protobacco ads and those with low receptivity to tobacco marketing, students exposed to any kind of pro-tobacco ads assessed in this study (convenient store, internet, newspaper/magazines, movies) and those with high receptivity to tobacco marketing were more curious about cigarettes. Finally, students who agree that all tobacco products are dangerous or believe people harm themselves when they smoke somedays were less curious about cigarette smoking (Table 1). 
Research Paper

Table 1. Descriptive statistics for a sample of youth who never tried cigarettes, National Youth Tobacco Survey, 2014

\begin{tabular}{|c|c|c|c|c|}
\hline Characteristic & $\mathbf{N}^{*}$ & $\begin{array}{c}\text { Susceptible to cigarettes } \\
\qquad \%(95 \% \text { CI }) S\end{array}$ & $\mathbf{N}^{*}$ & $\begin{array}{l}\text { Curious about cigaretles } \\
\qquad \%(95 \% \text { CI }) g\end{array}$ \\
\hline \multicolumn{5}{|l|}{ Sociodemographic } \\
\hline $\begin{array}{l}\text { Sex } \\
\text { Girls } \\
\text { Boys }\end{array}$ & $\begin{array}{l}8,310 \\
8,243\end{array}$ & $\begin{array}{l}26.83(25.38-28.33) \\
28.38(26.99-29.81)\end{array}$ & $\begin{array}{l}8,308 \\
8,236\end{array}$ & $\begin{array}{l}30.73(29.21-32.30) \\
29.26(27.67-30.89)\end{array}$ \\
\hline $\begin{array}{l}\text { Age in years } \\
\leq 13 \\
14 \\
15 \\
16 \\
\geq 17\end{array}$ & $\begin{array}{l}7,132 \\
2,590 \\
2,122 \\
1,940 \\
2,807\end{array}$ & $\begin{array}{l}26.75(25.05-28.51) \\
30.57(27.10-34.27) \\
30.77(28.20-33.46) \\
27.75(25.43-30.20) \\
24.16(21.51-27.03)\end{array}$ & $\begin{array}{l}7,127 \\
2,583 \\
2,127 \\
1,941 \\
2,804\end{array}$ & $\begin{array}{l}28.13(26.85-29.45) \\
34.40(31.30-37.65) \\
33.32(31.08-35.64) \\
29.13(26.64-31.75) \\
28.26(25.71-30.95)\end{array}$ \\
\hline $\begin{array}{l}\text { Race/ethnicity } \\
\text { White } \\
\text { Black } \\
\text { Hispanic } \\
\text { Other/multiple race }\end{array}$ & $\begin{array}{l}6,813 \\
2,453 \\
4,530 \\
2,054\end{array}$ & $\begin{array}{l}25.93(24.58-27.34) \\
26.40(24.17-28.76) \\
34.19(32.47-35.96) \\
26.40(23.30-29.75)\end{array}$ & $\begin{array}{l}6,814 \\
2,458 \\
4,524 \\
2,048\end{array}$ & $\begin{array}{l}29.22(27.94-30.54) \\
26.77(24.41-29.27) \\
34.28(32.39-36.23) \\
33.15(30.38-36.05)\end{array}$ \\
\hline $\begin{array}{l}\text { Grade } \\
\text { Middle school } \\
\text { High school }\end{array}$ & $\begin{array}{l}8,991 \\
7,589\end{array}$ & $\begin{array}{l}27.48(25.69-29.34) \\
27.73(26.13-29.38)\end{array}$ & $\begin{array}{l}8,979 \\
7,591\end{array}$ & $\begin{array}{l}29.10(27.87-30.35) \\
30.91(29.14-32.74)\end{array}$ \\
\hline Current use of tobacco/ni & & & & \\
\hline $\begin{array}{l}\text { Current waterpipe smok } \\
\text { No } \\
\text { Yes }\end{array}$ & $\begin{array}{c}15,839 \\
367\end{array}$ & $\begin{array}{l}26.99(25.87-28.13) \\
48.46(43.08-53.88)\end{array}$ & $\begin{array}{l}16,255 \\
390\end{array}$ & $\begin{array}{l}29.79(28.68-30.93) \\
42.64(36.56-48.96)\end{array}$ \\
\hline $\begin{array}{l}\text { Current electronic ciga } \\
\text { No } \\
\text { Yes }\end{array}$ & $\begin{array}{c}15,952 \\
517\end{array}$ & $\begin{array}{l}26.50(25.38-27.66) \\
60.50(55.21-65.56)\end{array}$ & $\begin{array}{l}15,947 \\
516\end{array}$ & $\begin{array}{l}29.37(28.24-30.52) \\
50.74(44.59-56.86)\end{array}$ \\
\hline $\begin{array}{l}\text { Current cigar/cigarillo/ } \\
\text { No } \\
\text { Yes }\end{array}$ & $\begin{array}{c}16,151 \\
232\end{array}$ & $\begin{array}{l}27.33(26.17-28.52) \\
42.72(35.44-50.32)\end{array}$ & $\begin{array}{c}16,144 \\
230\end{array}$ & $\begin{array}{l}30.03(28.88-31.20) \\
32.66(25.08-41.26)\end{array}$ \\
\hline $\begin{array}{l}\text { Current smokeless toba } \\
\text { No } \\
\text { Yes }\end{array}$ & $\begin{array}{c}15,982 \\
162\end{array}$ & $\begin{array}{l}27.31(26.17-28.47) \\
40.10(32.18-48.58)\end{array}$ & $\begin{array}{c}15,979 \\
162\end{array}$ & $\begin{array}{l}30.14(29.01-31.29) \\
28.30(21.53-36.22)\end{array}$ \\
\hline Exposure to secondhand si & & & & \\
\hline $\begin{array}{l}\text { Live now with waterpip } \\
\text { No } \\
\text { Yes }\end{array}$ & $\begin{array}{c}16,264 \\
391\end{array}$ & $\begin{array}{l}27.22(26.04-28.43) \\
45.08(38.89-51.43)\end{array}$ & $\begin{array}{c}16,255 \\
390\end{array}$ & $\begin{array}{l}29.69(28.52-30.88) \\
46.47(40.25-52.80)\end{array}$ \\
\hline $\begin{array}{l}\text { Live now with cigarette } \\
\text { No } \\
\text { Yes }\end{array}$ & $\begin{array}{l}12,575 \\
4,080\end{array}$ & $\begin{array}{l}25.78(24.55-27.04) \\
33.41(31.47-35.41)\end{array}$ & $\begin{array}{c}12,570 \\
4,075\end{array}$ & $\begin{array}{l}28.60(27.20-30.04) \\
34.66(32.63-36.75)\end{array}$ \\
\hline $\begin{array}{l}\text { Live now with electroni } \\
\text { No } \\
\text { Yes }\end{array}$ & $\begin{array}{c}15,518 \\
1,137\end{array}$ & $\begin{array}{l}26.75(25.53-28.00) \\
39.50(36.11-42.99)\end{array}$ & $\begin{array}{c}15,514 \\
1,131\end{array}$ & $\begin{array}{l}29.47(28.29-30.67) \\
38.09(34.29-42.04)\end{array}$ \\
\hline Exposure to pro-tobacco o & keting & & & \\
\hline $\begin{array}{l}\text { See cigarette ads at gas s } \\
\text { Never } \\
\text { Rarely/sometimes } \\
\text { Most of the time/always }\end{array}$ & $\begin{array}{c}966 \\
5,507 \\
9,202\end{array}$ & $\begin{array}{l}19.74(16.65-23.24) \\
26.29(24.60-28.05) \\
28.91(27.63-30.22)\end{array}$ & $\begin{array}{c}967 \\
5,502 \\
9,198\end{array}$ & $\begin{array}{l}20.67(17.58-24.14) \\
28.55(26.89-30.27) \\
32.20(30.93-33.49)\end{array}$ \\
\hline $\begin{array}{l}\text { See actors using cigare } \\
\text { Never } \\
\text { Rarely/sometimes } \\
\text { Most of the time/always }\end{array}$ & $\begin{array}{c}1,318 \\
10,153 \\
4,369\end{array}$ & $\begin{array}{l}23.53(20.60-26.73) \\
27.28(26.03-28.57) \\
29.92(27.85-32.08)\end{array}$ & $\begin{array}{c}1,319 \\
10,143 \\
4,368\end{array}$ & $\begin{array}{l}22.47(19.62-25.60) \\
30.19(28.92-31.49) \\
32.64(30.28-35.08)\end{array}$ \\
\hline $\begin{array}{l}\text { See cigarette ads in nev } \\
\text { Never } \\
\text { Rarely/sometimes } \\
\text { Most of the time/always }\end{array}$ & $\begin{array}{l}1,864 \\
7,984 \\
1,563\end{array}$ & $\begin{array}{l}22.41(19.85-25.21) \\
29.22(27.82-30.67) \\
26.88(23.37-30.70)\end{array}$ & $\begin{array}{l}1,859 \\
7,977 \\
1,561\end{array}$ & $\begin{array}{l}24.54(21.95-27.34) \\
31.95(30.53-33.40) \\
31.87(29.66-34.17)\end{array}$ \\
\hline $\begin{array}{l}\text { See cigarette ads when } \\
\text { Never } \\
\text { Rarely/sometimes } \\
\text { Most of the time/always }\end{array}$ & $\begin{array}{c}2,543 \\
11,097 \\
2,117\end{array}$ & $\begin{array}{l}19.65(17.64-21.82) \\
29.15(27.93-30.41) \\
28.23(25.55-31.06)\end{array}$ & $\begin{array}{c}2,544 \\
11,098 \\
2,109\end{array}$ & $\begin{array}{c}22.21(20.03-24.56) \\
32.09(30.90-33.30) \\
29.44(26.96-32.06)\end{array}$ \\
\hline
\end{tabular}




\begin{tabular}{|c|c|c|c|c|}
\hline \multicolumn{5}{|c|}{ Receptivity to tobacco marketing } \\
\hline Low & 14,639 & $25.63(24.40-26.89)$ & 14,641 & $28.58(27.44-29.75)$ \\
\hline High & 1,394 & $52.22(49.31-55.12)$ & 1,388 & $49.52(46.35-52.69)$ \\
\hline \multicolumn{5}{|c|}{ Beliefs about harm due to smoking } \\
\hline \multicolumn{5}{|c|}{ Agree that 'All tobacco products are dangerous' } \\
\hline Disagree & 1,265 & $40.66(37.83-43.55)$ & 1,269 & $37.23(34.05-40.52)$ \\
\hline Agree & 14,641 & $26.63(25.50-27.78)$ & 14,632 & $29.41(28.24-30.62)$ \\
\hline \multicolumn{5}{|c|}{$\begin{array}{l}\text { Believe how much people harm themselves when } \\
\text { smoke somedays, but not everyday }\end{array}$} \\
\hline No harm & 404 & 43.74 (37.74-49.93) & 402 & $33.29(27.74-39.34)$ \\
\hline Little/some harm & 6,715 & $36.68(35.03-38.36)$ & 6,710 & $39.27(37.50-41.06)$ \\
\hline A lot of harm & 9,147 & $20.33(19.21-21.50)$ & 9,146 & $23.28(22.17-24.44)$ \\
\hline
\end{tabular}

* Unweighted frequency of all youth who never smoked cigarettes, not even a puff or two. The number of missing values is unequal in each of the two studied outcomes. $\S$ Weighted row percent of susceptible youth. TWeighted row percent of curious youth.

Table 2. Multivariable logistic regression for factors associated with susceptibility and curiosity about cigarettes among youth who never tried cigarettes, National Youth Tobacco Survey, 2014

\begin{tabular}{|c|c|c|c|c|c|c|}
\hline \multirow[t]{2}{*}{ Characteristic } & \multicolumn{3}{|c|}{ Susceptibility to cigarettes } & \multicolumn{3}{|c|}{ Curiosity about cigarettes } \\
\hline & aOR & $95 \% \mathrm{CI}$ & p value & $\mathrm{aOR}$ & $95^{\circ} \mathrm{CI}$ & p value \\
\hline \multicolumn{7}{|l|}{ Sociodemographic } \\
\hline $\begin{array}{l}\text { Sex } \\
\text { Girls } \\
\text { Boys }\end{array}$ & $\begin{array}{l}\text { Ref } \\
0.98\end{array}$ & $0.86-1.11$ & 0.78 & $\begin{array}{l}\text { Ref } \\
0.86\end{array}$ & $0.74-0.99$ & 0.04 \\
\hline $\begin{array}{l}\text { Race/ethnicity } \\
\text { White } \\
\text { Black } \\
\text { Hispanic } \\
\text { Other/multiple race }\end{array}$ & $\begin{array}{l}\text { Ref } \\
0.99 \\
1.59 \\
1.15\end{array}$ & $\begin{array}{c}0.82-1.20 \\
1.40-1.80 \\
0.92-1.44\end{array}$ & $\begin{array}{c}0.95 \\
<0.0001 \\
0.19\end{array}$ & $\begin{array}{l}\text { Ref } \\
0.96 \\
1.33 \\
1.30\end{array}$ & $\begin{array}{l}0.81-1.14 \\
1.19-1.48 \\
1.10-1.54\end{array}$ & $\begin{array}{c}0.71 \\
<0.0001 \\
0.002\end{array}$ \\
\hline \multicolumn{7}{|c|}{ Current use of tobacco/nicotine products } \\
\hline $\begin{array}{l}\text { Current waterpipe smoking } \\
\text { No } \\
\text { Yes }\end{array}$ & $\begin{array}{l}\text { Ref } \\
1.52\end{array}$ & $1.01-2.26$ & 0.04 & $\begin{array}{l}\text { Ref } \\
1.19\end{array}$ & $0.83-1.70$ & 0.33 \\
\hline $\begin{array}{l}\text { Current electronic cigarett } \\
\text { No } \\
\text { Yes }\end{array}$ & $\begin{array}{l}\text { Ref } \\
3.67\end{array}$ & $2.76-4.86$ & $<0.0001$ & $\begin{array}{c}\text { Ref } \\
2.18\end{array}$ & $1.58-2.99$ & $<0.0001$ \\
\hline $\begin{array}{l}\text { Current smokeless toba } \\
\text { No } \\
\text { Yes }\end{array}$ & $\begin{array}{l}\text { Ref } \\
0.94\end{array}$ & $0.48-1.82$ & 0.86 & $\begin{array}{l}\text { Ref } \\
0.44\end{array}$ & $0.24-0.78$ & 0.006 \\
\hline Exposure to secondhand sn & & & & & & \\
\hline $\begin{array}{l}\text { Live now with waterpipe si } \\
\text { No } \\
\text { Yes }\end{array}$ & $\begin{array}{l}\text { Ref } \\
1.51\end{array}$ & $1.06-2.14$ & 0.02 & $\begin{array}{c}\text { Ref } \\
1.37\end{array}$ & $0.97-1.93$ & 0.06 \\
\hline $\begin{array}{l}\text { Live now with cigarette } \\
\text { No } \\
\text { Yes }\end{array}$ & $\begin{array}{l}\text { Ref } \\
1.24\end{array}$ & $1.08-1.42$ & $<0.002$ & $\begin{array}{l}\text { Ref } \\
1.15\end{array}$ & $0.98-1.34$ & 0.07 \\
\hline Exposure to pro-tobacco a & marketi & & & & & \\
\hline $\begin{array}{l}\text { See pro-tobacco ads in ne } \\
\text { Never } \\
\text { Rarely/sometimes } \\
\text { Most of the time/always }\end{array}$ & $\begin{array}{l}\text { Ref } \\
1.31 \\
1.16\end{array}$ & $\begin{array}{l}1.08-1.57 \\
0.89-1.51\end{array}$ & $\begin{array}{c}0.005 \\
0.25\end{array}$ & $\begin{array}{l}\text { Ref } \\
1.23 \\
1.30\end{array}$ & $\begin{array}{l}1.03-1.46 \\
1.03-1.65\end{array}$ & $\begin{array}{l}0.02 \\
0.03\end{array}$ \\
\hline $\begin{array}{l}\text { See pro-tobacco ads in the } \\
\text { Never } \\
\text { Rarely/sometimes } \\
\text { Most of the time/always }\end{array}$ & $\begin{array}{l}\text { Ref } \\
1.24 \\
1.15\end{array}$ & $\begin{array}{l}1.01-1.54 \\
0.89-1.47\end{array}$ & $\begin{array}{l}0.04 \\
0.26\end{array}$ & $\begin{array}{l}\text { Ref } \\
1.28 \\
1.11\end{array}$ & $\begin{array}{l}1.05-1.55 \\
0.86-1.41\end{array}$ & $\begin{array}{l}0.01 \\
0.39\end{array}$ \\
\hline $\begin{array}{l}\text { Receptivity to tobacco ma } \\
\text { Low } \\
\text { High }\end{array}$ & $\begin{array}{l}\text { Ref } \\
2.81\end{array}$ & $2.40-3.30$ & $<0.0001$ & $\begin{array}{l}\text { Ref } \\
2.18\end{array}$ & $1.83-2.60$ & $<0.0001$ \\
\hline
\end{tabular}




\begin{tabular}{|c|c|c|c|c|c|c|}
\hline \multicolumn{7}{|c|}{ Beliefs about harm due to smoking } \\
\hline \multicolumn{7}{|c|}{ Agree that 'All tobacco products are dangerous' } \\
\hline Disagree & Ref & & & Ref & & \\
\hline Agree & 0.88 & $0.72-1.07$ & 0.22 & 0.80 & $0.65-0.98$ & 0.03 \\
\hline \multicolumn{7}{|c|}{$\begin{array}{l}\text { Believe how much people harm themselves } \\
\text { when smoke somedays, but not everyday }\end{array}$} \\
\hline No harm & Ref & & & Ref & & \\
\hline Little/some harm & 0.74 & $0.40-1.36$ & 0.33 & 1.32 & $0.89-1.95$ & 0.15 \\
\hline A lot of harm & 0.29 & $0.16-0.54$ & $<0.0001$ & 0.63 & $0.42-0.942$ & 0.02 \\
\hline
\end{tabular}

\section{Multivariable analysis}

Table 2 presents results from multivariable logistic regression models assessing the association between current waterpipe smoking, and curiosity and susceptibility to cigarette smoking. 1) Current waterpipe use and susceptibility to cigarette smoking Among students who reported never smoked cigarettes, current waterpipe smokers were 1.5 times more susceptible to cigarette smoking than non-current waterpipe smokers; controlling for socio-demographics, use of other combustible and non-combustible tobacco products, receptivity to tobacco marketing, beliefs and attitudes towards tobacco use, and exposure to pro-tobacco ads $(\mathrm{aOR}=1.52,95 \%$ CI: $1.01-2.26$, $\mathrm{p}=0.04$ ). Students living with someone who smokes waterpipe $(\mathrm{aOR}=1.51,95 \%$ CI: $1.06-2.14, \mathrm{p}=0.02)$, or cigarettes ( $\mathrm{aOR}=1.24,95 \%$ CI: $1.08-1.42, \mathrm{p}<0.002$ ) were more likely to be susceptible to cigarette smoking.

2) Use of other tobacco products, exposure to tobacco ads and susceptibility to cigarette smoking

Electronic cigarette users were 3.6 times more likely than non-users to be susceptible to cigarette smoking ( $\mathrm{aOR}=3.6$, 95\% CI: $2.76-4.86, \mathrm{p}<0.0001)$. Those reported exposure to pro-tobacco ads rarely/sometimes in newspapers/magazines $(\mathrm{aOR}=1.31,95 \%$ CI: $1.08-1.57, \mathrm{p}=0.005)$, or the internet $(\mathrm{aOR}=1.24,95 \%$ CI: 1.01-1.54, $\mathrm{p}=0.04)$, and those with high receptivity to tobacco marketing ( $\mathrm{aOR}=2.81,95 \% \mathrm{CI}$ : $2.40-3.30, \mathrm{p}<0.0001$ ) were more likely to be susceptible to cigarette smoking. Finally, the belief that people harm themselves a lot when they smoke somedays was protective against susceptibility to cigarettes $(\mathrm{aOR}=0.29,95 \%$ CI: 0.16 $0.54, \mathrm{p}<0.0001)$.

3) Current waterpipe use and curiosity about cigarette smoking Current waterpipe smoking was not associated with curiosity about cigarettes $(\mathrm{aOR}=1.19,95 \%$ CI: $0.83-1.70, \mathrm{p}=0.33$ ). A borderline association was observed between curiosity about cigarettes and living with someone who smokes waterpipe $(\mathrm{aOR}=1.37,95 \%$ CI: $0.97-1.93, \mathrm{p}=0.06)$ or cigarettes ( $\mathrm{aOR}=1.15,95 \%$ CI: 0.98-1.34, $\mathrm{p}=0.07$ ).

4) Use of other tobacco products, exposure to tobacco ads and curiosity about cigarette smoking

Electronic cigarette users were more curious about cigarettes
$(\mathrm{aOR}=2.18,95 \%$ CI: $1.58-2.99, \mathrm{p}<0.0001)$ than non-users, while smokeless tobacco users were less curious about cigarettes $(\mathrm{aOR}=0.44,95 \%$ CI: $0.24-0.78, \mathrm{p}=0.006)$. Higher odds of curiosity about cigarettes were associated with exposure to pro-tobacco ads in newspaper/magazines rarely/sometimes ( $\mathrm{aOR}=1.23,95 \% \mathrm{CI}: 1.03-1.46, \mathrm{p}=0.02)$, or most of the times/ always $(\mathrm{aOR}=1.30,95 \% \mathrm{CI}: 1.03-1.65, \mathrm{p}=0.03)$, exposure to pro-tobacco ads rarely/sometimes on the internet $(\mathrm{aOR}=1.28$, 95\% CI: $1.05-1.55, \mathrm{p}=0.01$ ), and high receptivity to tobacco marketing ( $\mathrm{aOR}=2.18,95 \%$ CI: $1.83-2.60, \mathrm{p}<0.0001)$. The belief that all tobacco products are dangerous $(\mathrm{aOR}=0.80,95 \%$ CI: $0.65-0.98, p=0.03$ ), and that people harm themselves a lot when they smoke somedays $(\mathrm{aOR}=0.63,95 \%$ CI: $0.42-0.942$, $\mathrm{p}=0.02$ ) were protective against curiosity about cigarettes. (Table 2).

\section{DISCUSSION}

Using a representative sample of cigarette-naïve US youth, it was found that $48.5 \%$ of current waterpipe smokers and $45.1 \%$ of those living with a waterpipe smoker were susceptible to cigarette smoking, compared with $27 \%$ among non-current waterpipe smokers and $27.2 \%$ among those not living with a waterpipe smoker. Current waterpipe smokers and those living with a waterpipe smoker were 1.5 times more susceptible to cigarettes than those not smoking waterpipe or living with a waterpipe smoker. Although a higher proportion of current waterpipe smokers and those living with a waterpipe smoker were curious about cigarettes, these associations did not reach statistical significance. These findings suggest that cigarettenaive youth who use waterpipe are highly susceptible to cigarettes, but not more curious about cigarettes, which may support the underlying assumption of the gateway hypothesis that increasing demand to nicotine may lead to cigarette smoking among cigarette-naïve adolescents who smoke waterpipe ${ }^{10}$.

The finding that waterpipe smoking was associated with susceptibility but not curiosity deserves further discussion. This is probably due to the differences in defining each concept. Curiosity is measured directly by asking participants if they were ever curious about cigarettes, while susceptibility is measured indirectly using a composite index of 3 items: 
how likely is it that participants will smoke a cigarette in the next year; will try a cigarette soon; and the self-efficacy to refuse a cigarette offered by a close friend. Another plausible explanation is that youths who smoke waterpipe might initiate cigarettes as a result of increased susceptibility to cigarettes, perhaps due to increased demand for nicotine, rather than an increasing curiosity about cigarettes. This needs to be tested in a longitudinal study.

Previous studies have shown that waterpipe use among youth who never smoked can lead to initiation of cigarette smoking $^{9-11}$. To the best of the author's knowledge, this study is the first to show that waterpipe use is associated with increased susceptibility to cigarette smoking using a large, representative sample of cigarette-naïve US youth. A possible explanation for this observation is that youth who smoke waterpipe may be more susceptible to initiating cigarette smoking to satisfy a craving for nicotine by using the easily accessible cigarette, compared to a less mobile, less accessible waterpipe ${ }^{10}$. Another finding of this study is that living with a waterpipe smoker is associated with a higher susceptibility to cigarette smoking. It is well established that exposure to secondhand smoke is associated with higher susceptibility and subsequent initiation of cigarette smoking among cigarettenaïve youth ${ }^{23-25}$. Youth living with a smoker possibly perceive smoking as a socially acceptable practice that can encourage initiation of smoking. Although the US has adopted indoor air policies against smoking, paradoxically, waterpipe is exempt from such policies ${ }^{26}$. Extending indoor air polices to include waterpipe and addressing exposure to waterpipe tobacco smoking at home can be effective in preventing susceptibility and initiation of cigarettes among cigarette-naïve US youth.

Although marketing restrictions on tobacco ads and promotions have been in place since 1998, many neversmoking US youth are still exposed to pro-tobacco ads and at risk of smoking initiation ${ }^{27}$. This study shows that exposure to pro-tobacco ads mainly on the internet and in newspapers/ magazines was associated with curiosity and susceptibility to cigarette smoking among youth who never smoked. In fact, US middle and high school students are increasingly exposed to pro-tobacco ads on the internet ${ }^{28}$. This finding is of great concern given that waterpipe ads on the internet are less likely, compared with cigarette ads, to depict smoking in a negative way, mention the harmful effects of smoking, show antismoking messages, or provide advice on quitting ${ }^{29}$. In addition, less than $1 \%$ of the waterpipe marketplace on the internet shows any warning against tobacco on the first web page, and many use deceptive descriptions of waterpipe appealing to youth ${ }^{30}$. More importantly, unregulated pro- tobacco ads expose cigarette-naïve youth to smoking images that can normalize smoking ${ }^{15,29}$.

This study highlights important demographic disparities in the susceptibility and curiosity about cigarettes that have important policy implications. First, Hispanic youth were more susceptible and curious about cigarettes than nonHispanic white youth. This is likely because Hispanic youth have lower perceived harm of tobacco use and at higher risk of initiating smoking ${ }^{31}$. Second, boys were less curious about cigarettes than girls in this study. While concerns about weight control may explain why young girls were more curious about cigarettes than boys, other factors such as boys' higher curiosity about alternative tobacco products, cigars and smokeless tobacco as examples, may explain this observation $^{32}$.

This study has limitations. Tobacco use was self-reported and subject to recall errors. However, estimates were based on current tobacco use, which provides more valid estimates of tobacco use among youth ${ }^{33}$. Given the cross-sectional nature of NYTS, a causal inference between waterpipe use and susceptibility to cigarettes cannot be established. Strengths include the large sample size, a good overall response rate of $73.3 \%$ achieved in NYTS $2014^{6}$, and the generalizability of these findings to middle and high school students in the US.

\section{CONCLUSIONS}

In summary, this study presents data on the association between current waterpipe use, and susceptibility and curiosity about cigarettes among a nationally representative sample of US youth. Current waterpipe use or living with a waterpipe user was independently associated with susceptibility, but not curiosity, to cigarette smoking. These findings suggest that experimentation with cigarettes among cigarette-naïve adolescent waterpipe smokers can be due to increasing susceptibility, where the more addicted youths may need to use cigarettes to satisfy nicotine craving rather than having higher curiosity to use cigarettes. This needs to be examined in a longitudinal study.

\section{REFERENCES}

1. U.S. Department of Health and Human Services. Preventing Tobacco Use Among Youth and Young Adults: A Report of the Surgeon General. Atlanta, GA: U.S. Department of Health and Human Services, Centers for Disease Control and Prevention, National Center for Chronic Disease Prevention and Health Promotion, Office on Smoking and Health, 2012.

2. Grucza RA, Bierut LJ. Cigarette smoking and the risk for alcohol use disorders among adolescent drinkers. Alcohol Clin Exp Res 2006;30(12):2046-54.

doi: 10.1111/j.1530-0277.2006.00255.x 
3. CDC. Youth and Tobacco Use. Available at: https://www.cdc.gov/ tobacco/data_statistics/fact_sheets/youth_data/tobacco_use/index. htm (accessed Oct 18, 2017).

4. Maziak W, Taleb ZB, Bahelah R, Islam F, Jaber R, Auf R, Salloum RG. The global epidemiology of waterpipe smoking. Tob Control 2015;24 Suppl 1:i3-i12. doi: 10.1136/tobaccocontrol-2014-051903.

5. Arrazola RA, Singh T, Corey CG, Husten CG, Neff LJ, Apelberg BJ, Bunnell RE, Choiniere CJ, King BA, Cox S, McAfee T, Caraballo RS; Centers for Disease Control and Prevention (CDC). Tobacco use among middle and high school students - United States, 2011-2014. MMWR Morb Mortal Wkly Rep 2015;64(14):381-5.

6. Meier EM, Tackett AP, Miller MB, Grant DM, Wagener TL. Which nicotine products are gateways to regular use? First-tried tobacco and current use in college students. Am J Prev Med 2015;48(1 Suppl 1):S86-93. doi: 10.1016/j.amepre.2014.09.018

7. Gilreath TD, Leventhal A, Barrington-Trimis JL, Unger JB, Cruz TB, Berhane K, Huh J, Urman R, Wang K, Howland S, Pentz MA, Chou CP, McConnell R. Patterns of Alternative Tobacco Product Use: Emergence of Hookah and E-cigarettes as Preferred Products Amongst Youth. J Adolesc Health 2016;58(2):181-5. doi: 10.1016/j.jadohealth.2015

8. Maziak W. The waterpipe: a new way of hooking youth on tobacco. Am J Addict 2014;23(2):1037. doi: 10.1111/j.1521-0391.2013.12073.x

9. Soneji S, Sargent JD, Tanski SE, Primack BA. Associations between initial water pipe tobacco smoking and snus use and subsequent cigarette smoking: results from a longitudinal study of US adolescents and young adults. JAMA Pediatr 2015;169(2):129-36. doi: 10.1001/jamapediatrics.2014.2697

10. Jaber R, Madhivanan P, Veledar E, Khader Y, Mzayek F, Maziak W. Waterpipe a gateway to cigarette smoking initiation among adolescents in Irbid, Jordan: a longitudinal study. Int J Tuberc Lung Dis 2015;19(4):481-7. doi: 10.5588/ijtld.14.0869

11. Veeranki SP, Alzyoud S, Kheirallah KA, Pbert L. Waterpipe Use and Susceptibility to Cigarette Smoking Among Never-Smoking Youth. Am J Prev Med 2015;49(4):502-11. doi: 10.1016/j.amepre.2015.03.035

12. Nodora J, Hartman SJ, Strong DR, et al. Curiosity predicts smoking experimentation independent of susceptibility in a US national sample. Addict Behav 2014;39(12):1695-700. doi: 10.1016/j.addbeh.2014.06.002

13. Dube SR, Arrazola RA, Lee J, Engstrom M, Malarcher A. Protobacco influences and susceptibility to smoking cigarettes among middle and high school students--United States, 2011. J Adolesc Health 2013;52(5 Suppl):S45-51.

14. Persoskie A, Donaldson EA, King BA. Ever-Use and Curiosity About Cigarettes, Cigars, Smokeless Tobacco, and Electronic Cigarettes Among US Middle and High School Students, 2012-2014. Prev Chronic Dis 2016;13:E134.

15. Bunnell RE, Agaku IT, Arrazola RA, et al. . Intentions to smoke cigarettes among never-smoking US middle and high school electronic cigarette users: National Youth Tobacco Survey, 20112013. Nicotine Tob Res 2015;17(2):228-35.
16. CDC. National Youth Tobacco Survey (NYTS). Available from: http://www.cdc.gov/tobacco/data_statistics/surveys/nyts/ [Accessed Mar 18, 2017].

17. Portnoy DB, Wu CC, Tworek C, Chen J, Borek N. Youth curiosity about cigarettes, smokeless tobacco, and cigars: prevalence and associations with advertising. Am J Prev Med 2014;47(2 Suppl 1):S76-86.

doi: 10.1016/j.amepre.2014.04.012.

18. Veeranki SP, Mamudu HM, Anderson JL, Zheng S. Worldwide never-smoking youth susceptibility to smoking. J Adolesc Health 2014;54(2):144-50.

doi: 10.1016/j.jadohealth.2013.07.036.

19. CDC. Smokeless Tobacco: Products and Marketing. Available from: http://www.cdc.gov/tobacco/data_statistics/fact_sheets/ smokeless/products_marketing/index.htm [Accessed May 1, 2017].

20. Amrock SM, Gordon T, Zelikoff JT, Weitzman M. Hookah use among adolescents in the United States: results of a national survey. Nicotine Tob Res 2014;16(2):231-7. doi: 10.1093/ntr/ntt160.

21. Gilpin EA, Pierce JP. The California Tobacco Control Program and potential harm reduction through reduced cigarette consumption in continuing smokers. Nicotine Tob Res 2002;4 Suppl 2:S157-66. doi:10.1080/1462220021000032708.

22. Hosmer D, Lemeshow S. Applied Logistic Regression (Second Edition), 2000. New York: John Wiley \& Sons, Inc.

23. Lee KA, Palipudi KM, English LM, Ramanandraibe N, Asma S; GYTS collaborative group. Secondhand smoke exposure and susceptibility to initiating cigarette smoking among never-smoking students in selected African countries: Findings from the Global Youth Tobacco Survey. Prev Med 2016.

24. Agaku IT, Maliselo T, Ayo-Yusuf OA. The relationship between secondhand smoke exposure, pro-tobacco social influences, and smoking susceptibility among nonsmoking Zambian adolescents. Subst Use Misuse 2015;50(3):387-93.

25. Veeranki SP, Mamudu HM, Zheng S, John RM, Cao Y, Kioko D, Anderson J, Ouma AE. Secondhand smoke exposure among neversmoking youth in 168 countries. J Adolesc Health 2015;56(2):16773.

26. Jawad M, El Kadi L, Mugharbil S, Nakkash R. Waterpipe tobacco smoking legislation and policy enactment: a global analysis. Tob Control 2015;24 Suppl 1:i60-i65.

27. Fulmer EB, Neilands TB, Dube SR, Kuiper NM, Arrazola RA, Glantz SA. Protobacco Media Exposure and Youth Susceptibility to Smoking Cigarettes, Cigarette Experimentation, and Current Tobacco Use among US Youth. PLoS One 2015;10(8):e0134734. doi: 10.1371/journal.pone.0134734.

28. Agaku IT, King BA, Dube SR. Trends in exposure to pro-tobacco advertisements over the Internet, in newspapers/magazines, and at retail stores among U.S. middle and high school students, 20002012. Prev Med 2014;58:45-52. doi: 10.1016/j.ypmed.2013.10.012.

29. Carroll MV, Shensa A, Primack BA. A comparison of cigarette- and hookah-related videos on YouTube. Tob Control 2013;22(5):31923.

doi: 10.1136/tobaccocontrol-2011-050253.

30. Primack BA, Rice KR, Shensa A, Carroll MV, DePenna EJ, Nakkash R, Barnett TE. U.S. hookah tobacco smoking establishments

advertised on the internet. Am J Prev Med 2012;42(2):150-6. 


\section{Research Paper}

doi: 10.1016/j.amepre.2011.10.013.

31. El-Toukhy S, Choi K. Smoking-Related Beliefs and Susceptibility Among United States Youth Nonsmokers. J Adolesc Health 2015;57(4):448-50.

32. Portnoy DB, Wu CC, Tworek C, Chen J, Borek N. Youth curiosity about cigarettes, smokeless tobacco, and cigars: prevalence and associations with advertising. Am J Prev Med 2014;47(2 Suppl 1):S76-86.

33. Dolcini MM, Adler NE, Lee P, Bauman KE. An assessment of the validity of adolescent self-reported smoking using three biological indicators. Nicotine Tob Res 2003;5(4):473-83.

CONFLICT OF INTERESTS

The author has completed and submitted the ICMJE

Form for Disclosure of Potential Conflicts of Interest and none was reported.

\section{FUNDING}

There was no source of funding for this research.

PROVENANCE AND PEER REVIEW

Not commissioned;

externally peer reviewed 